\title{
Effects of galacto-oligosaccharides on growth and gut function of newborn suckling piglets
}

Shiyi Tian, Jue Wang, Hu Yu, Jing Wang ${ }^{*}$ and Weiyun Zhu

\begin{abstract}
Background: Most research on galacto-oligosaccharides (GOS) has mainly focused on their prebiotic effects on the hindgut, but their beneficial effects on the small intestine (SI) have received little attention. Since jejunum is the important place to digest and absorb nutrients efficiently, optimal maturation of the jejunum is necessary for maintaining the high growth rate in the neonate. Therefore, this study investigates the effect of the early intervention with GOS on the intestinal development of the jejunum.

Methods: A total of 6 litters of neonatal piglets (10 piglets per litter; Duroc $\times$ Landrace $\times$ Large White) with an average birth weight of $1.55 \pm 0.05 \mathrm{~kg}$ received 1 of 2 treatments based on their assignment to either the control (CON) group or the GOS (GOS) group in each litter. Piglets in the GOS group were orally administrated $10 \mathrm{~mL}$ of a GOS solution (reaching $1 \mathrm{~g} \mathrm{GOS} / \mathrm{kg}$ body weight) per day from the age of 1 to $7 \mathrm{~d}$; the piglets in the CON group were treated with the same dose of physiological saline. All piglets were weaned on $\mathrm{d} 21$. On $\mathrm{d} 8$ and 21 of the experimental trial, 1 pig per group from each of the 6 litters was euthanized.
\end{abstract}

Results: The early intervention with GOS increased the average daily gains in the third week $(P<0.05)$. Decreased crypt depth was also observed in the jejunum of the piglets on d $21(P<0.05)$. The early intervention with GOS increased the jejunal lactase activity on $d 8$, maltase activity and sucrase activity on $d 21(P<0.05)$. In addition, the early intervention with GOS also facilitated the mRNA expression of Sodium glucose co-transporter 1 (SGLT1) on d 8 and the mRNA expression of Glucose transporter type 2 (GLUT2) on d $21(P<0.05)$. It was further determined that GOS up-regulated the mRNA expression of preproglucagon (GCG), insulin-like growth factor 1 (IGF-1), insulin-like growth factor 1 receptor (IGF-1R) and epidermal growth factor (EGF). GOS also up-regulated the protein expression of glucagon-like peptide-2 (GLP-2) and EGF in the jejunum of the piglets. Furthermore, it was also found that GOS enhanced the protein expression of ZO-1 and occludin on d $8(P<0.05)$, as well as increased the mRNA expression of TGF- $\beta$ and decrease the mRNA expression of $I L-12(P<0.05)$.

Conclusions: These results indicate that GOS have a positive effect on piglet growth performance in addition to decreasing the crypt depth and enhancing functional development in jejunum of suckling piglets.

Keywords: Early intervention, Galacto-oligosaccharides, Growth performance, Intestinal development, Jejunum, Suckling piglets

\footnotetext{
* Correspondence: jwang8@njau.edu.cn

National Center for International Research on Animal Gut Nutrition, Jiangsu Key Laboratory of Gastrointestinal Nutrition and Animal Health, Laboratory of Gastrointestinal Microbiology, College of Animal Science and Technology, Nanjing Agricultural University, Nanjing 210095, China
}

(c) The Author(s). 2018 Open Access This article is distributed under the terms of the Creative Commons Attribution 4.0 International License (http://creativecommons.org/licenses/by/4.0/), which permits unrestricted use, distribution, and reproduction in any medium, provided you give appropriate credit to the original author(s) and the source, provide a link to the Creative Commons license, and indicate if changes were made. The Creative Commons Public Domain Dedication waiver (http://creativecommons.org/publicdomain/zero/1.0/) applies to the data made available in this article, unless otherwise stated. 


\section{Background}

A nutritional strategy to improve the growth performance of newborn animals is the basis of improving the overall productivity of animals [1]. The jejunum is an important part of the intestine in which the efficient digestion and absorption of nutrients take place, and the maturation of the jejunum is therefore beneficial for maintaining a high rate of growth among neonates $[2,3]$. During the neonatal period, the gastrointestinal tract of piglets develops rapidly [4]. Dietary nutrients are essential for gastrointestinal (GI) growth and functional development, and the nutritional support of GI growth and development is a significant part of the nursing process [5].

In recent years, probiotics and prebiotics have gained considerable attention as growth promoters. Galacto-oligosaccharides (GOS), a type of prebiotics, contain 2-8 saccharide units, where one of these units is terminal glucose and the remaining are galactoses and disaccharides comprised of 2 units of galactoses [6, 7]. GOS are attractive food additives for infant formula because of their capability of modulating the intestinal microbiota, improving intestinal development, enhancing mineral absorption, and protecting the intestinal barrier $[8,9]$. Previous studies have shown that GOS could promote the growth of beneficial bacteria and improve host health in vitro and in vivo [10-12]. However, the promotion of intestinal development and the enhancement of intestinal barrier properties by GOS have been described mainly in vitro using cell models such as the human Caco- 2 cell line and rodent models $[13,14]$; very little has been shown about the effects on suckling piglets in vivo. Moreover, most in vivo studies of GOS have been specifically restricted to the cecum and colon but very few data are currently available for the jejunum. It is essential to explore the effect of early intervention with GOS on the jejunal development of suckling piglets.

We hypothesized that early intervention with GOS could increase the growth performance and improve the intestinal development of suckling piglets. The present study was conducted to explore the effects of early intervention with orally administrated GOS on the jejunal morphology, digestive and absorptive functions, and barrier functions of suckling piglets.

\section{Methods}

\section{Animals and experimental treatments}

A total of 6 litters of neonatal piglets (10 piglets in each; Duroc $\times$ Landrace $\times$ Large White) were used in this study with an average birth weight of $1.55 \pm 0.05 \mathrm{~kg}$. Piglets from each litter were equally assigned to either the control (CON) group or the GOS (GOS) group to receive 1 of 2 treatments in order to account for any maternal differences. GOS-90S was obtained from Quantum Hi-Tech Biological Co., Ltd. (China), which contained oligosaccharides with a degree of polymerization (DP) of 2-8 with approximately 90\% (w/w) GOS, 8.5\% (w/ $w)$ lactose, and $1.5 \%(w / w)$ glucose on dry matter (DM). During the $7 \mathrm{~d}$ after birth, all piglets in the GOS group were orally administered $10 \mathrm{~mL}$ galacto-oligosaccharides (GOS) solution (1 g GOS/kg body weight $[9,10,15,16])$ per day. Half of the dose was given at 9:00 and the other at 18:00. Similarly, all the piglets in the CON group were orally administered the same dose of physiological saline. The solution was infused into each piglet's mouth by an injector without a needle with a soft infusion tube with a length of $5 \mathrm{~cm}$. To avoid the potential stress after the swallowing, the piglets were put in the nursing pen immediately. All piglets were weaned on d 21. The piglets had free access to sow milk and water at all times throughout the experimental period. Average bodyweight throughout the treatment process was recorded on $\mathrm{d} 7,14$ and 21 . Health status was monitored daily until $21 \mathrm{~d}$ of age, and all piglets remained healthy during the experimental period.

Sample collection and measurement of villus morphology On $\mathrm{d} 8$ and 21 of the experimental trial, 1 pig per group from each of the 6 litters was euthanized with an intravenous overdose of pentobarbital via a catheterized ear vein as described by Moeser et al. [17]. Blood samples were collected from the anterior vena cava into tubes containing sodium heparin and immediately mixed to avoid coagulation. Plasma was obtained after centrifugation at $3,000 \times g$ for $15 \mathrm{~min}$ at $4{ }^{\circ} \mathrm{C}$ and then stored at $80{ }^{\circ} \mathrm{C}$ until analysis. The small intestine (SI) was also removed. The length of the SI was measured after stripping off the mesentery by holding the intestine vertically against a ruler. The wet weight of the SI was determined after gently squeezing out the intestinal contents and removing the mesentery and fat.

The mid-jejunal samples (midpoint between the pylorus and the ileocecal valve) were preserved in a formaldehyde and glutaraldehyde mixing fixative. Then the cross-sections of mid-jejunal samples were prepared using standard paraffin embedding techniques. To measure villus height and crypt depth, the samples were sectioned at $6 \mu \mathrm{m}$ thickness and stained with hematoxylin and eosin (HE) [18]. Mucosal samples from the proximal jejunum (at $40 \mathrm{~cm}$ from the duodenum-jejunum junction) were collected using a glass slide, rapidly frozen in liquid nitrogen, and stored at $-80{ }^{\circ} \mathrm{C}$ for further analysis. All samples were collected within $20 \mathrm{~min}$ after the piglet had been euthanized. The total protein in the mucosal was extracted according to the instructions of a total protein extraction kit (KGP2100; Keygen Biotech, Nanjing, China). The mucosal protein concentration of the supernatant fractions was then quantified by a 
standard bicinchoninic acid (BCA) protein assay (A0453, JianCheng Bioengineering Institute, Nanjing, Jiangsu, China).

\section{RNA extraction, cDNA synthesis, and real-time RT-PCR for gene expression analysis}

The frozen jejunal mucosa was homogenized in $1 \mathrm{~mL}$ Trizol Reagent (Invitrogen, Carlsbad, CA, USA), and the total RNA was isolated according to the manufacture's recommendations. The absorption ratio $(260 / 280 \mathrm{~nm})$ of all the samples was between 1.8 and 2.0, which indicated high purity of the RNA. The total RNA was reverse-transcribed to cDNA using a PrimeScript RT reagent kit with a gDNA eraser (Takara Biotechnology (Dalian) Co., Ltd.) according to the recommended procedures.

The primers for the intestinal nutrient transporter genes: sodium glucose co-transporter 1 (SGLT1), glucose transporter type 2 (GLUT2); the intestinal growth factors: preproglucagon $(G C G)$, insulin-like growth factor 1 (IGF-1), insulin-like growth factor 1 receptor (IGF-1R), epidermal growth factor $(E G F)$; the intestinal barrier related genes: zonula occludens-1 (ZO-1), occludin; the intestinal immune factors: interleukin-1 $\beta(I L-1 \beta)$, interleukin-10 (IL-10), interleukin-12 (IL-12), toll-like receptor 4. (TLR4), transforming growth factor- $\beta$ (TGF- $\beta)$, tumor necrosis factor- $\alpha(T N F-\alpha)$ and housekeeping genes $(\beta$-actin and glyceraldehyde phosphate dehydrogenase $(G A P D H))$ are listed in Additional file 1: Table S1.

The target genes and housekeeping genes were measured with an Applied Biosystems 7300 Real-Time PCR system using a SYBR Premix Ex Taq (Tli RnaseH Plus) qPCR kit (Takara Biotechnology (Dalian) Co., Ltd.) according to the manufacturer's guidelines. The standard dilution and samples were assayed in triplicate in a $20 \mu \mathrm{L}$ reaction mixture containing $10 \mu \mathrm{L}$ of SYBR, $0.4 \mu \mathrm{L} 0.2 \mu \mathrm{mol} / \mathrm{L}$ of forward and reverse primer, $6.8 \mu \mathrm{L}$ nuclease-free water, and $2 \mu \mathrm{L}$ of $100 \mathrm{ng} / \mu \mathrm{L}$ DNA template. The cycling conditions were $95{ }^{\circ} \mathrm{C}$ for $30 \mathrm{~s}$, followed by 40 cycles of $95{ }^{\circ} \mathrm{C}$ for $5 \mathrm{~s}$ and $60{ }^{\circ} \mathrm{C}$ for $34 \mathrm{~s}$. The standard curve was also included in each run to determine PCR efficiency. The specificity and efficiency of the selected primers were confirmed by qRT-PCR analysis and a dilution series of pooled cDNA at a temperature gradient $\left(55-65{ }^{\circ} \mathrm{C}\right)$ for primer-annealing and subsequent melting curve analysis. The stability of the housekeeping genes was evaluated by measuring the fluctuation range of the $\mathrm{Ct}$ values (Ct values were obtained by real-time quantitative PCR). Then, the two candidate genes were analyzed by NormFinder software [19]. The $\beta$-actin was finally identified as the housekeeping gene because no variation in its expression was observed between treatments. The mRNA expression levels were calculated using the $2^{-\Delta \Delta \mathrm{Ct}}$ method [20]. All the data were normalized to those of the housekeeping gene $\beta$-actin. The CON group was then established as the control group. The relative expression of the target gene mRNA in each group was calculated as follows: $\Delta \mathrm{Ct}=\mathrm{Ct}$ (target gene) - $\mathrm{Ct}(\beta$-actin $)$, and $\Delta \Delta \mathrm{Ct}=\Delta \mathrm{Ct}$ (treated group) $-\Delta \mathrm{Ct}$ (CON group on $\mathrm{d} 8$ ).

\section{$D$-lactic acid and diamine oxidase}

The levels of diamine oxidase (DAO; Enzyme Commission Number (EC) 1.4.3.6) and D-lactic acid were used as the indices of intestinal mucosal injury in the piglets. The levels of $D$-lactic acid in the plasma were determined with a $D$-lactic acid colorimetric assay kit (BioVision Inc., Milpitas, CA). $D$-lactic acid in the plasma was expressed in terms of $\mathrm{mg} / \mathrm{L}$. Diamine oxidase activities in the plasma and jejunal mucosa were measured using an enzymatic spectrophotometric assay as described by $\mathrm{Hu}$ et al. [21]. Diamine oxidase activities in the plasma and jejunal mucosa were expressed in terms of units $/ \mathrm{mL}$ and units/mg mucosa, respectively.

\section{Disaccharidase activity}

The enzymes studied here were lactase (EC 3.2.1.23), sucrase (EC 3.2.1.48) and maltase (EC 3.2.1.20). All assays were carried out on the homogenates of mucosal tissue obtained by thawing approximately $0.1 \mathrm{~g}$ of tissue and homogenizing it in $9 \mathrm{~mL}$ phosphate buffer saline (PBS, $\mathrm{pH}=7.2$ ) with an ultrasonic homogenizer. The homogenate was then centrifuged $\left(500 \times g, 10 \mathrm{~min}\right.$ at $\left.4{ }^{\circ} \mathrm{C}\right)$, and the supernatants were collected. The activity levels of the digestive enzymes lactase (Lactase Activity Testing Kit, No: A082-1), sucrase (Sucrase Activity Testing Kit, No: A082-2) and maltase (Maltase Activity Testing Kit, No: A082-3) were determined according to the instructions of the manufacturer Nanjing JianCheng Bioengineering Institute (Nanjing, Jiangsu, China).

\section{Intestinal growth factors}

The levels of intestinal growth factors (glucagon-like peptide-1 (GLP-1), glucagon-like peptide-2 (GLP-2), insulin-like growth factor 1 (IGF-1), and epidermal growth factor (EGF)) in the intestinal mucosa were determined using the ProcartaPlex ${ }^{\text {Ti }}$ multiplex immunoassay kit (Luminex, Austin, USA) according to the manufacturer's instructions obtained from Affymetrix eBioscience (Santa Clara, USA). The results were normalized against the total protein concentration for each sample in an inter-sample comparison.

\section{Tight junction protein expressions}

Tight junction protein expressions of zonula occludens-1 (ZO-1) and occludin were measured by Western blotting. After the protein concentration of supernatant fractions was quantified by a standard bicinchoninic acid (BCA) protein assay (Pierce, Rockford, IL, USA), the standardized 
protein amounts of boiled samples were isolated with a $15 \%$ sodium dodecyl sulfate-polyacrylamide gel electrophoresis (SDS-PAGE) and electro-transferred onto polyvinylidene difluoride (PVDF) membranes (Merck Millipore). Membranes were blocked in a skim milk TBS buffer $(15 \mathrm{mmol} / \mathrm{L}$ Tris- $\mathrm{HCl}, 150 \mathrm{mmol} / \mathrm{L} \mathrm{NaCl}$, $10 \%$ skim milk; pH 7.4) and incubated overnight at $4{ }^{\circ} \mathrm{C}$ with antibodies for occludin (1:1,000; Abcam) or ZO-1 (1:1,000; Invitrogen). After being washed in a phosphate buffer solution with Tween-20 (PBST), the membranes were incubated with an appropriate horseradish peroxidase-conjugated secondary antibody (1: 2,000; Fcmacs-Bio, Beijing, China) for $2 \mathrm{~h}$ at room temperature. Finally, the PVDF membranes harboring the target bands were visualized through an electrochemiluminescence system (Tanon, Shanghai, China). Subsequently, the membranes were re-probed with a $\beta$-actin antibody $(1: 2,000$; Cell Signaling) to assess the equality of loading. Band intensities were quantified using ImageJ version 1.47 software (National Institute of Health, American), and the protein expression was normalized with $\beta$-actin and expressed as the mean fold change in relation to the control group.

\section{Statistical analysis}

Data were analyzed by SPSS 20.0 (IBM, US) and expressed as means \pm SEM or means \pm SD. The model included the fixed effects of diet, age, associated interactions, and any random errors with respect to a group or an individual piglet. Bodyweight (BW) and average daily gain (ADG) were evaluated using the group in each litter as the experimental unit. The other parameters were assessed using each slaughtered piglet as an experimental unit. The data were evaluated by two-way ANOVA, and differences were considered significant at $P<0.05$. When a significant dietary effect or an interaction between diet and time was observed, the data were further analyzed by using one-way ANOVA with Duncan's post hoc test. And a value of $P<0.05$ was used to indicate statistical significance, whereas a $P$-value between 0.05 and 0.10 was considered to indicate a trend toward significance.

\section{Results}

\section{Growth performance and digestive organ indexes}

The effects of early intervention with GOS on the growth performance of suckling piglets are illustrated in Fig. 1 . BW was significantly $(P<0.05)$ affected by the age of piglets, and it also tended to be affected by diet $(P=$ 0.099). Piglets fed with diets containing GOS showed higher BW than those fed with no GOS on $d 21$, but the difference was not significant. For ADG, there was a significant interaction $(P<0.05)$ between diet and the age of the piglets. Piglets fed with diets containing GOS

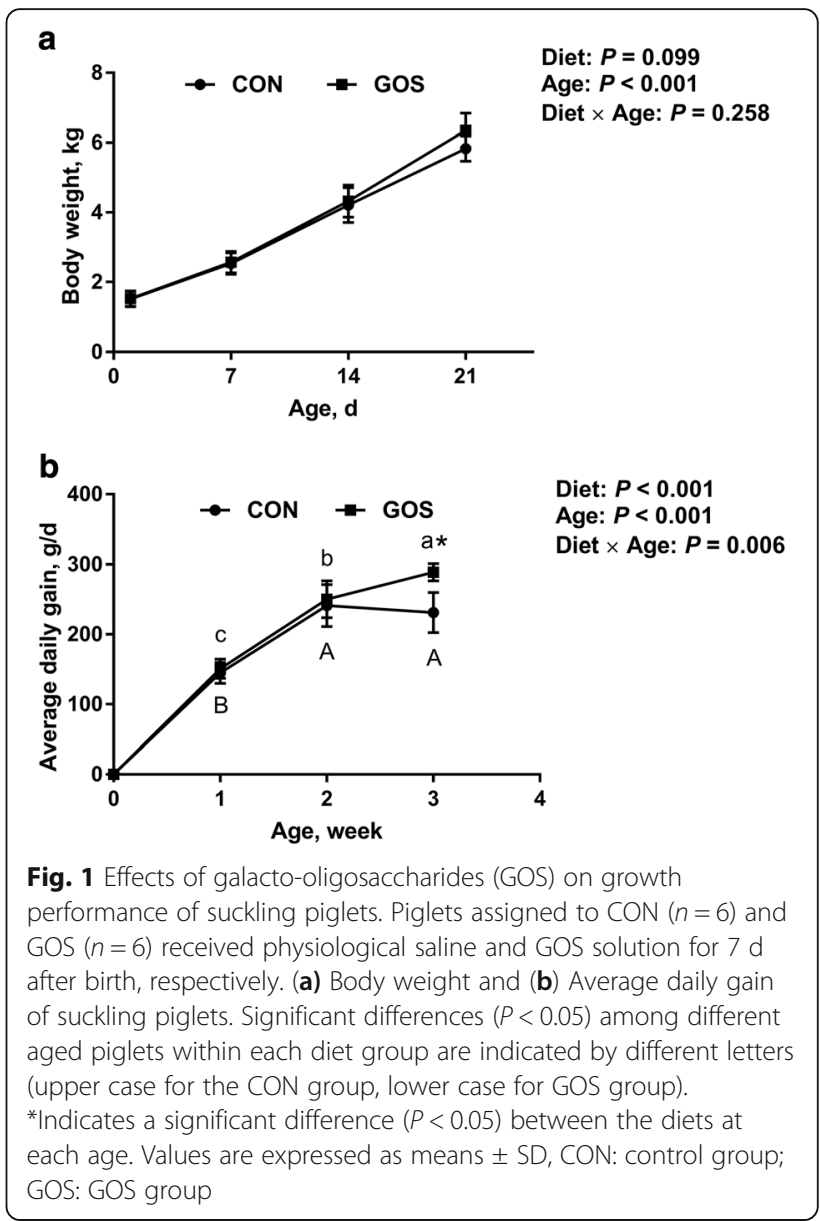

showed significantly higher ADG $(P<0.05)$ than those fed with no GOS in the third week. In addition, the results with respect to the digestive organs are presented in Table 1 . SI length was significantly affected by diet $(P$ $<0.05$ ), and the same tendency was reported for SI weight $(P=0.078)$; SI weight / BW tended to be affected by the interaction between diet and age $(P=0.096)$. Furthermore, piglets fed with diets containing GOS showed significantly increased SI length $(P<0.05)$ than those fed with no GOS on $\mathrm{d} 8$.

\section{Intestinal morphology and intestinal growth factors}

Diet and age had no significant interactive effects on villus height and the villus height / crypt depth ratio of the jejunum of piglets $(P>0.05)$. Crypt depth was significantly affected by the interaction between diet and age $(P<0.05)$. Piglets fed with diets containing GOS showed significantly less crypt depth $(P<0.05)$ than those fed with no GOS on d 21 (Fig. 2).

The effects of early intervention with GOS on the mRNA expression of intestinal growth factors are presented in Fig. 3a-d. The mRNA expression of IGF-1, $I G F-1 R$, and $E G F$ was affected by interactions between 
Table 1 Effects of galacto-oligosaccharides (GOS) on digestive organ indexes of suckling piglets ${ }^{\mathrm{a}}$

\begin{tabular}{|c|c|c|c|c|c|c|c|c|}
\hline \multirow[t]{2}{*}{ Items } & \multicolumn{2}{|l|}{ d 8} & \multicolumn{2}{|l|}{ d 21} & \multirow[t]{2}{*}{ SEM } & \multicolumn{3}{|c|}{$P$-value } \\
\hline & CON & GOS & CON & GOS & & Diet & Age & Diet $\times$ Age \\
\hline $\mathrm{Sl}^{\mathrm{b}}$ weight, $\mathrm{g}$ & 79.72 & 107.32 & 193.23 & 197.74 & 8.66 & 0.078 & $<0.001$ & 0.196 \\
\hline SI length, m & $4.39^{c}$ & $4.93^{b}$ & $6.64^{\mathrm{a}}$ & $6.81^{\mathrm{a}}$ & 0.17 & 0.041 & $<0.001$ & 0.290 \\
\hline SI weight/SI length, $\mathrm{g} / \mathrm{m}$ & 19.03 & 21.50 & 29.06 & 28.86 & 0.82 & 0.183 & $<0.001$ & 0.121 \\
\hline SI weight/BW, g/kg & 26.85 & 31.92 & 31.45 & 31.20 & 1.53 & 0.131 & 0.218 & 0.096 \\
\hline SI length/BW, m/kg & 1.43 & 1.49 & 1.08 & 1.08 & 0.04 & 0.553 & $<0.001$ & 0.473 \\
\hline
\end{tabular}

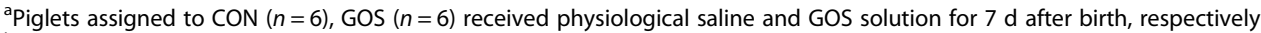

${ }^{b}$ SI small intestinal

diet and age $(P<0.05)$, and the mRNA expression of $G C G$ was affected by diet. Piglets fed with diets containing GOS showed significantly higher mRNA expression of GCG, IGF-1, IGF-1R and EGF than those fed with no GOS on $\mathrm{d} 8(P<0.05)$, and there was also a significantly higher mRNA expression of GCG in GOS-fed piglets than in those fed with no GOS on $\mathrm{d} 21(P<0.05)$.

The effects of early intervention with GOS on the protein expression of intestinal growth factors are shown in Fig. 3e-h. The results show that the protein expression of GLP-2 and EGF were affected by diet. Piglets fed with diets containing GOS showed significantly higher protein expression of GLP-2 and EGF than those fed with no $\operatorname{GOS}(P<0.05)$.

\section{Disaccharidase activity and nutrient transporters}

As shown in Fig. $4 \mathrm{a}-\mathrm{c}$, lactase and sucrase activity were affected by interactions between diet and age $(P<0.05)$, and maltase activity was affected by diet. More specifically, piglets fed with diets containing GOS showed significantly higher maltase and sucrase activities $(P<$ 0.05 ) than those fed with no GOS on $d 21$, while piglets fed with diets containing GOS showed significantly higher lactase activity $(P<0.05)$ than those fed with no GOS on d 8.

To assess gene expression related to the nutrient transporters of jejunum, two kinds of genes were assayed by q-PCR, as shown in Fig. 4d-e. It was demonstrated that the mRNA expression of SGLT1 and GLUT2 was affected by interactions between diet and age $(P<0.05)$. Piglets fed with diets containing GOS showed significantly higher mRNA expression of GLUT2 $(P<0.05)$ than those fed with no GOS on d 8, while piglets fed with diets containing GOS showed significantly higher mRNA expression of SGLT1 $(P<0.05)$ than those fed with no GOS on $\mathrm{d} 21$.

\section{$D$-lactic acid, diamine oxidase, tight junction genes, and protein expressions}

The plasma $D$-lactic acid concentration and DAO activity in the suckling pigs are presented in Table 2. Significant interactions between diet and age $(P<0.05)$ were observed in the plasma $D$-lactic acid concentration, plasma DAO, and jejunal mucosa DAO. On d 8, GOS significantly reduced plasma $D$-lactic acid concentration and DAO activity $(P<0.05)$, and increased jejunal mucosa DAO $(P<0.05)$.

Figure 5 shows the gene expression and protein expression of ZO-1 and occludin in the jejunal mucosa of the piglets. Significant dietary effects were observed on the occludin mRNA expression levels $(P<0.05)$, and a higher mRNA expression of occludin was observed in piglets fed with GOS $(P<0.05)$ in contrast to those fed with no GOS on d 8 (Fig. 5a-b). As shown in Fig. 5c-e, the protein expression of $\mathrm{ZO}-1$ and occludin was affected by interactions between diet and age $(P<0.05)$. The protein expression of ZO-1 and occludin in the jejunal mucosa also increased in the GOS group on $\mathrm{d} 8$ $(P<0.05)$. In addition, the protein expression of occludin in the jejunal mucosa of the GOS piglets was higher than that of the CON piglets.

\section{Intestinal immune factors}

With respect to intestinal immune factors (Fig. 6), no significant interactions between diet and age were observed on the mRNA expression of $I L-1 \beta, I L-10, T L R 4$ and TNF- $\alpha(P>0.05)$. However, significant interactions between diet and age were observed on the TGF- $\beta$ mRNA expression levels $(P<0.05)$, and significant dietary effects were observed on the IL-12 mRNA expression levels $(P<0.05)$. It was also determined that GOS increased the mRNA expression of TGF- $\beta(P<$ $0.05)$ and reduced the mRNA expression of $I L-12$ on $\mathrm{d} 8$ $(P<0.05)$.

\section{Discussion}

In the present study, a neonatal piglet model was used to study the effects of early intervention with GOS on growth performance and jejunal development during a week-long intervention period. By determining the effects of early feeding strategies on the entire suckling stage, the current experiment made it possible to evaluate the effects of GOS (from d 1 to d 7) on piglets' growth performance, jejunal morphology, disaccharidase 


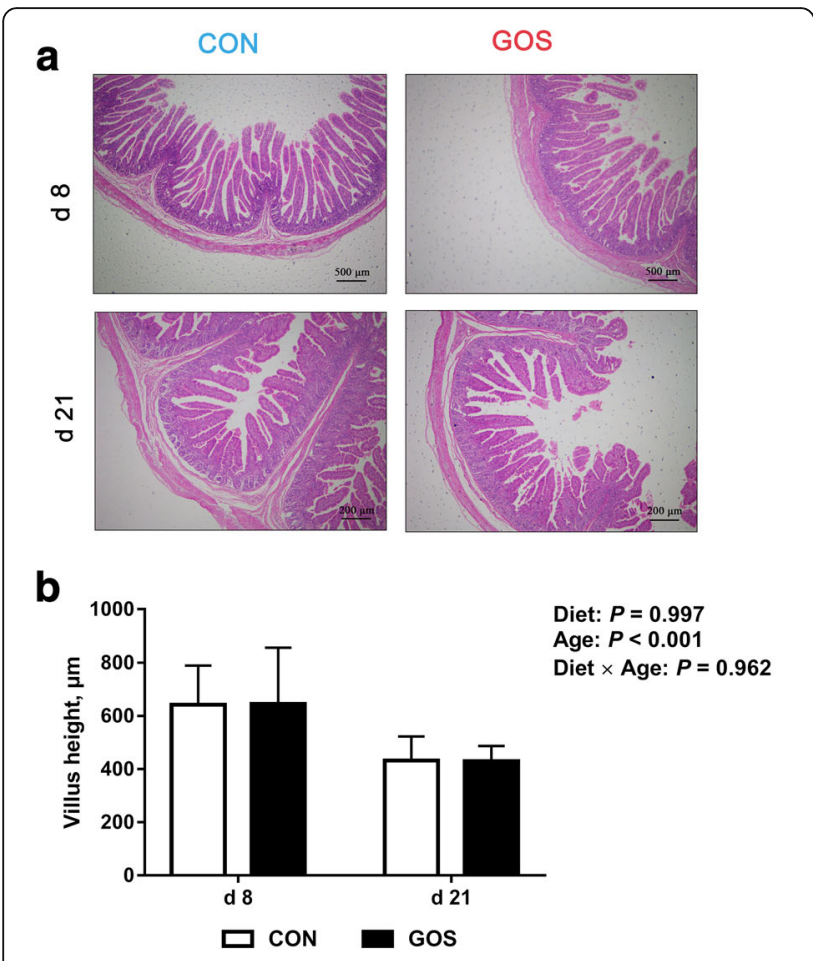

C

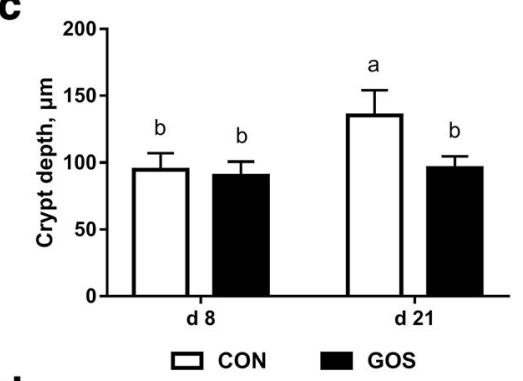

d

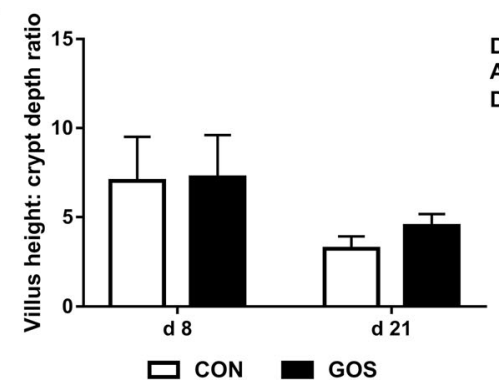

Fig. 2 The jejunal morphology of suckling piglets. Piglets assigned to $\operatorname{CON}(n=6)$ and $\operatorname{GOS}(n=6)$ received physiological saline and GOS solution for $7 \mathrm{~d}$ after birth, respectively. (a) Representative histological micrographs of jejunum in suckling piglets. The scale bar of jejunal morphology on d 8 was $500 \mu \mathrm{m}$, and the scale bar of jejunal morphology on d 21 was $200 \mu \mathrm{m}$. (b) Villus height, (c) Crypt depth and (d) Villus height: crypt depth radio of jejunal morphology in suckling piglets. Values are expressed as means \pm SD. Bars assigned with different lower-case letters indicate a significant difference. CON: control group; GOS: GOS group activity, and barrier function at different ages. The results suggested that GOS had significant effects on ADG, SI length, crypt depth, disaccharidase activity, tight junction expression, and gut permeability in suckling piglets.

In our study, we referred to the dose of GOS used in rodent model to determine the appropriate dose of GOS for piglets. In previous studies, only the effect of increased abundance of Bifidobacterium has been reported in mice fed GOS of $0.26 \mathrm{~g} /(\mathrm{kg} \cdot \mathrm{d})$ [10]. While increased cecal total weight and wall weight have been reported in mice fed GOS of $1 \mathrm{~g} /(\mathrm{kg} \cdot \mathrm{d})$ [16]. Anthony et al. [15] found that rats fed GOS of $2.5 \mathrm{~g} /(\mathrm{kg} \cdot \mathrm{d})$ or $5 \mathrm{~g} /(\mathrm{kg} \cdot \mathrm{d}) \mathrm{de}-$ creased food consumption levels. Based on the reported results, the dose of $1 \mathrm{~g} \mathrm{GOS} / \mathrm{kg}$ weight was administered in our study. In addition, it has been reported that the natural oligosaccharide content of sows is approximately in the range of $0.05-0.1 \mathrm{~g} / \mathrm{dL}$ [22]. According to the Alizadeh's [23] research, we estimated that the total oligosaccharide intake from the sow milk was about 0.3$0.6 \mathrm{~g} / \mathrm{d}$ when the piglets received sow milk of $600 \mathrm{~mL} / \mathrm{d}$ during the $7 \mathrm{~d}$ after birth. In our study, piglets eventually intake GOS of $1.37-2.31 \mathrm{~g} / \mathrm{d}$ (the initial BW of GOS group: $1.52 \mathrm{~kg}$, the BW on 7 of GOS group: $2.57 \mathrm{~kg}$ ) with a consideration of the purity of GOS, about 4 times higher than the oligosaccharide intake solely from sow milk by the piglets.

Previous studies have shown that the small intestine has demonstrated a significant increase in tissue mass and surface area of absorption in neonatal piglets [24-28]. For instance, the number of mucosal cells reportedly increased by $50 \%$ on the first day after birth and doubled on the third day after birth [28]. These studies indicated that the intestine of piglets developed fastest in the early stages of suckling piglets. Furthermore, it has been reported that GOS could increase cecal total weight and wall weight in mice [16]. Therefore, the purpose of this study was to improve intestinal development and increase the growth performance in the early stages of suckling piglets by supplementing with GOS. Consistent with our purpose, the SI length was significantly increased in GOS group on d 8. The increased SI length indicated that a significant increase in the area of the digestive and absorption of nutritious substances, thereby improving the growth performance of piglets. In addition, there have been several attempts to demonstrate the use of GOS as potential promoters to enhance animal growth $[29,30]$. Along the same lines as these studies, we have observed that early intervention with GOS could improve the BW and ADG of suckling piglets, which also consistent with our purpose.

For suckling piglets, intestinal growth factors play a key role in the development of the intestine. For example, the GLP-1, GLP-2, EGF, and IGF-1 proteins 

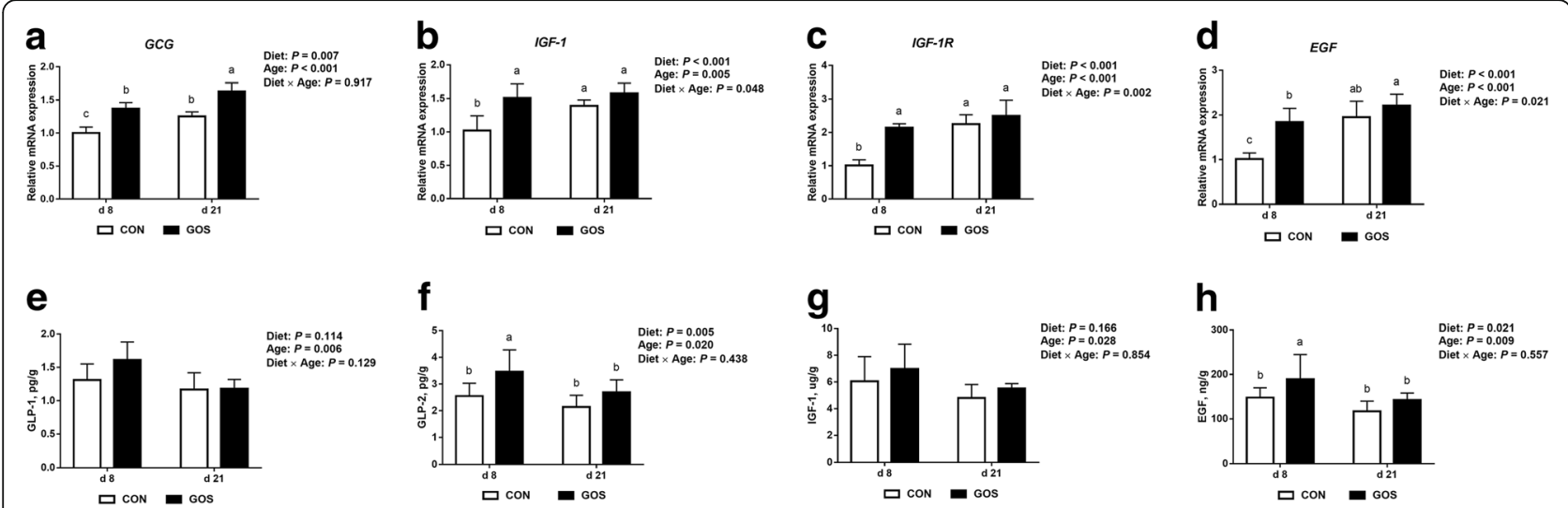

Fig. 3 The expression of jejunal growth factors in suckling piglets. Piglets assigned to $\operatorname{CON}(n=6)$ and $\operatorname{GOS}(n=6)$ received physiological saline and GOS solution for $7 \mathrm{~d}$ after birth, respectively. a-d The relative mRNA expression of jejunal growth factors in suckling piglets. The values were calculated relative to the expression of $\beta$-actin with formula $2^{-\Delta \Delta c t}$. e-h The concentrations of jejunal growth factors in suckling piglets. Values are expressed as means \pm SD. Bars assigned with different lower-case letters indicate significant differences. CON: control group; GOS: GOS group

were able to increase the proliferation, differentiation, and apoptosis of intestinal epithelial cells [31, 32]. In this study, we observed that the expressions of the intestinal growth factors differed between GOS and CON groups on $\mathrm{d} 8$, but not on $\mathrm{d} 21$. But interestingly, the expressions of intestinal growth factors in GOS piglets on $\mathrm{d} 8$ were close to those in $\mathrm{CON}$ piglets and GOS piglets on d 21. According to previous results, the concentrations of growth factors in sow milk at early lactation stage are higher than those at the late lactation stage. This may cause the growth and development of the jejunum to reach the plateau stage at the late period of lactation [33]. And our dynamic change of daily weight gain confirms this speculation. Furthermore, we also observed that the mRNA expression of IGF-1 and GCG (the precursor of glucagon and other components is encoded by the GCG) in the GOS group was higher than that in $\mathrm{CON}$ group, and the protein concentration of GLP-2 was consistent with the mRNA expression of the GCG. The increased mRNA and protein expression of GLP-2 could increase SI length through the stimulation of epithelial cell antiapoptotic actions by activators of the PI3K-Akt pathway [31]. The activation of Akt in the intestinal mucosa has also been implicated in GLP-2-mediated epithelial glucose uptake [31]. In addition, IGF-1 has been identified as a major mediator through which GLP-2 increases intestinal growth [34]. Also, high mRNA and protein expression of GLP-2 may also be modulated by nutrient intake, especially carbohydrate intake $[35,36]$. Therefore, we believe that GOS could improve the growth performance of suckling piglets via promoting jejunal development and increasing carbohydrate intake.
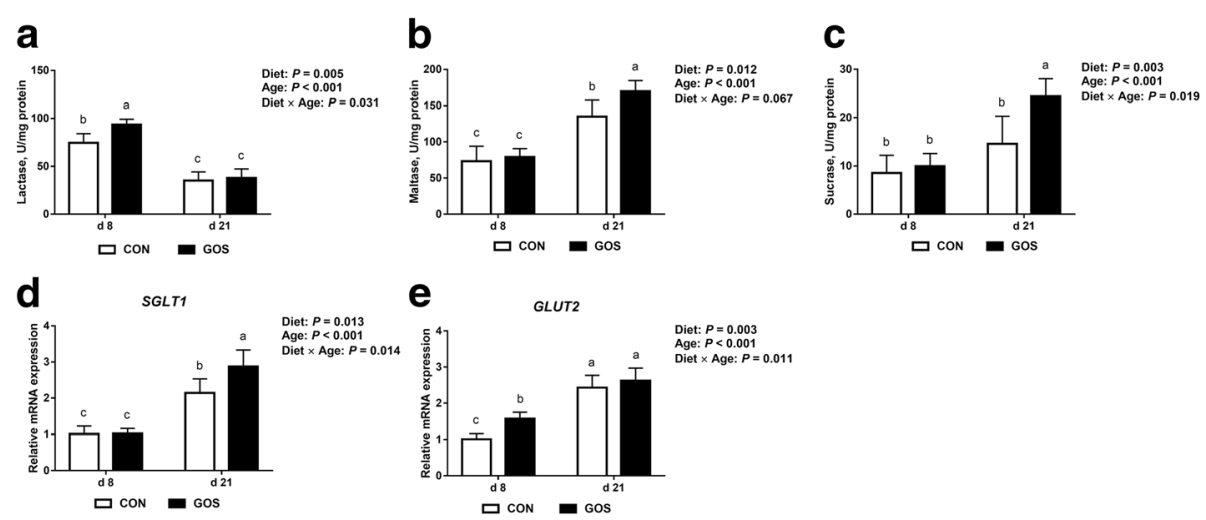

Fig. 4 The jejunal disaccharidase activity and mRNA expression of the glucose transport receptors in suckling piglets. Piglets assigned to CON $(n=6)$ and GOS $(n=6)$ received physiological saline and GOS solution for $7 \mathrm{~d}$ after birth, respectively. (a-c) The brush border enzyme activity of the jejuna in suckling piglets. (d-e) The relative mRNA expression of jejunal nutrient transporter in suckling piglets. The values were calculated relative to the expression of $\beta$-actin with formula $2^{-\Delta \Delta C t}$. Values are expressed as means $\pm S D$. Bars assigned with different lower-case letters indicate significant differences. CON: control group; GOS: GOS group 
Table 2 Effects of galacto-oligosaccharides (GOS) on D-lactic acid and diamine oxidase (DAO) in suckling piglets ${ }^{a}$.

\begin{tabular}{|c|c|c|c|c|c|c|c|c|}
\hline \multirow[t]{2}{*}{ Items } & \multicolumn{2}{|l|}{ d 8} & \multicolumn{2}{|l|}{ d 21} & \multirow[t]{2}{*}{ SEM } & \multicolumn{3}{|c|}{$P$-value } \\
\hline & CON & GOS & CON & GOS & & Diet & Age & Diet $\times$ Age \\
\hline Plasma D-lactic acid, mg/L & $13.13^{\mathrm{a}}$ & $12.42^{b}$ & $12.62^{b}$ & $12.81^{\mathrm{ab}}$ & 0.15 & 0.116 & 0.661 & 0.010 \\
\hline Plasma DAO, units/mL & $3.67^{\mathrm{a}}$ & $3.26^{\mathrm{b}}$ & $3.24^{b}$ & $3.25^{b}$ & 0.08 & 0.019 & 0.008 & 0.012 \\
\hline Jejunal mucosa DAO, units/mg mucosa & $2.18^{b}$ & $2.32^{a}$ & $2.20^{b}$ & $2.21^{\mathrm{b}}$ & 0.03 & 0.017 & 0.170 & 0.025 \\
\hline
\end{tabular}

${ }^{a}$ Piglets assigned to CON $(n=6), \operatorname{GOS}(n=6)$ received physiological saline and GOS solution for $7 \mathrm{~d}$ after birth, respectively

Since the jejunum is the main organ for nutrient absorption, we further analyzed jejunal morphology, disaccharidase activity, and carbohydrate transporters. In the present study, early intervention with GOS significantly decreased the crypt depth on $\mathrm{d} 21$, but it did not affect the villus height and villus height: crypt depth ratio in the jejunum. The most direct factor affecting crypt depth was the change in the proliferation rate of intestinal stem cells [37]. In addition, decreased crypt depth indicated that cell proliferation had decreased in the GOS group. Villus height has been positively correlated with the number of cells present [37]. In this study, there was no difference in the villus height between the two groups, which indicated that decreased cell proliferation did not affect the growth of the jejunum. This may have been caused by the increased cell differentiation and the decreased cell apoptosis of the jejunum. Furthermore, disaccharidase activity is related to intestinal morphology. And the disaccharidase activity determines suckling piglets' capacity for carbohydrate digestion and transport. In previous studies, lactase activity was high at birth but decreased with the age. However, sucrase activity and maltase activity were low at birth, but their activity gradually increased with the age until reaching stability [38]. Consistent with the findings of these studies, the lactase activity on $\mathrm{d} 21$ was lower than that on $\mathrm{d} 8$, and the sucrase and maltase activities on $\mathrm{d} 21$ were higher than those on $\mathrm{d} 8$. It is known that maltase and sucrase activities are important markers to evaluate intestinal development $[39,40]$. Hence, the increase of maltase and sucrase activities implied a certain rapid maturation of the jejunum. In addition, the present study showed that early intervention with GOS up-regulated lactase activity on $\mathrm{d} 8$, and maltase, sucrase activities on $\mathrm{d}$ 21 . Furthermore, the up-regulated lactase activity on $\mathrm{d} 8$, and the maltase, sucrase activities on $\mathrm{d} 21$ would promote the polysaccharides in sow milk to be degraded into monosaccharides. This is conducive to the body absorbing and utilizing the carbohydrates, thereby promoting intestinal maturity and host growth. These results therefore suggested that the piglets in the GOS group could utilize carbohydrates more efficiently than those in the CON group. After hydrolyzation by disaccharidase, the carbohydrates in a diet depend on a carbohydrate transporter to enter the bloodstream. In this study, the mRNA expressions of SGLT1 and GLUT2 were higher in the piglets with GOS intervention than those in the piglets without GOS intervention, indicating an increased glucose transport rate of the intestine. Overall, these results indicate that early intervention with GOS enhances the degradation

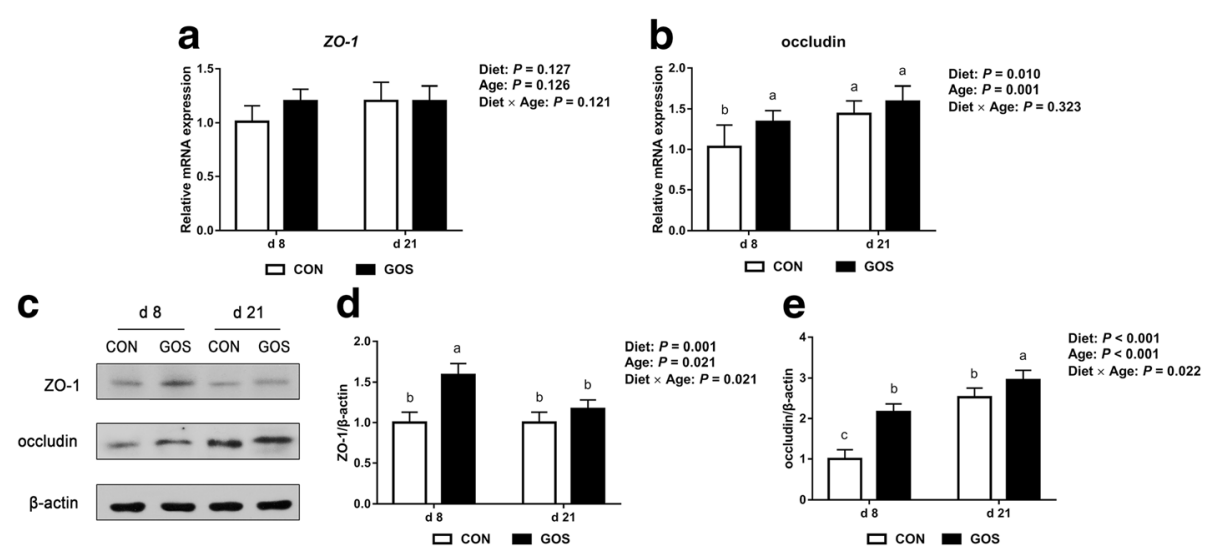

Fig. 5 The relative mRNA and protein expression of the jejunal tight junction in suckling piglets. Piglets assigned to CON $(n=6)$ and GOS $(n=6)$ received physiological saline and GOS solution for $7 \mathrm{~d}$ after birth, respectively. (a-b) The relative mRNA expression of the jejunal tight junction in suckling piglets. The values were calculated relative to the expression of $\beta$-actin with formula $2^{-\triangle \Lambda C t}$. (c) The blots of zonula occludens-1 (ZO-1), occludin, and $\beta$-actin of the jejunum mucosa in suckling piglets. (d-e) The relative protein expressions of the jejunal tight junction in suckling piglets. The value of protein expression was the ratio of the densitometry units of tight junction protein to $\beta$-actin. Values are expressed as means \pm SD. Bars assigned with different lower-case letters indicate significant differences. CON: control group; GOS: GOS group 

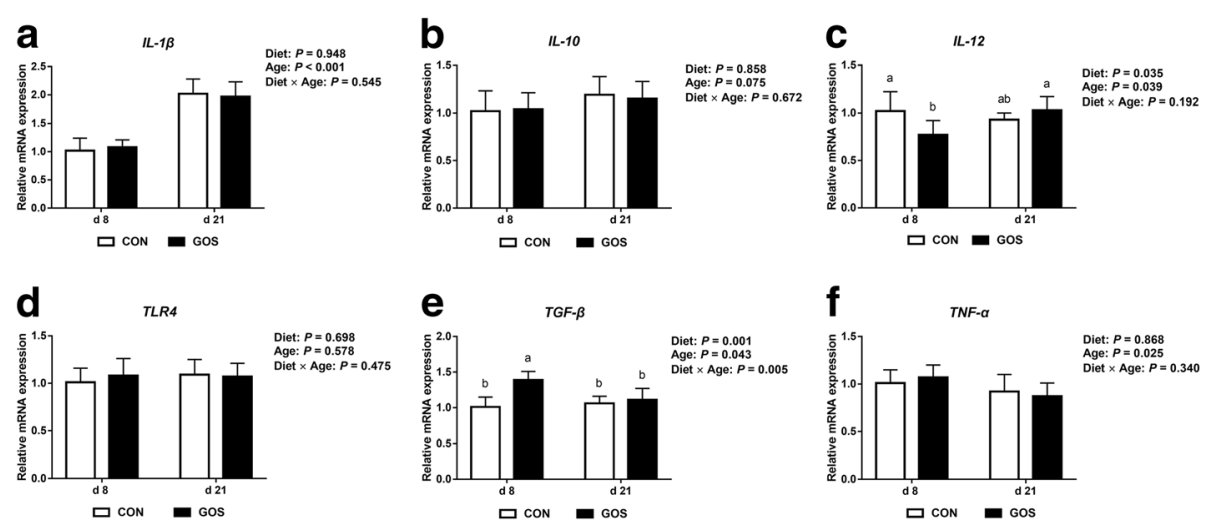

Fig. 6 The jejunal immune function in suckling piglets. Piglets assigned to CON $(n=6)$ and $\operatorname{GOS}(n=6)$ received physiological saline and GOS solution for $7 \mathrm{~d}$ after birth, respectively. (a-f) The relative mRNA expression of jejunal immune factors in suckling piglets. The values were calculated relative to the expression of $\beta$-actin with formula $2^{-\Delta \Delta C t}$. Values are expressed as means \pm SD. Bars assigned with different lower-case letters indicate significant differences. CON: control group; GOS: GOS group

rate of carbohydrates and the glucose transport rate in suckling piglets by modulating disaccharidase activity and the expression of glucose transport receptors.

A good mechanical barrier can effectively prevent bacteria, endotoxins, and other harmful substances from penetrating the intestinal mucosa, and in terms of infrastructure, it functions as a tight junction between the intact intestinal epithelial cells and other epithelial cells [41-43]. ZO-1 and occludin are main transmembrane and nonmembrane proteins that form intercellular junctions between the epithelial cells $[44,45]$. In addition, DAO and $D$-lactate serve as indicators of intestinal integrity, as they are normally presented in very small amounts in blood circulation. Increased plasma $D$-lactic acid levels and serum DAO levels reflect changes in intestinal permeability, suggesting that the intestinal barrier function has been damaged [46, 47]. Many studies have shown that GOS can reduce gut permeability and increase tight junction expression in vivo and in vitro $[48,49]$. Consistent with previous research results, our study shows that early intervention with GOS could improve the protein expression of $\mathrm{ZO}-1$ and occludin in the jejunal mucosa on d 8. We also observed that plasma $D$-lactate and DAO decreased in the GOS group on $\mathrm{d} 8$. These results indicated that early intervention with GOS could enhance the barrier function of the jejunum. Furthermore, the improvement of intestinal barrier function may imply the improvement of intestinal immune function. Therefore, we analyzed the mRNA expression of inflammatory factors. In this study, GOS increased the mRNA expression of TGF- $\beta$ and reduced the mRNA expression of $I L-12$ on $\mathrm{d}$ 8. IL-12 is a pro-inflammatory cytokine, and TGF- $\beta$ is an anti-inflammatory cytokine. According to the results, early
GOS intervention promoted the maturation of immune function. In addition, several studies have shown that the strength of the intestinal barrier was associated with enhanced piglet performance [43, 50]. In our study, the improvement of intestinal barrier function was accompanied by increased growth performance. Therefore, increased barrier function may ensure the absorption of nutrients and prevent bacteria, endotoxins, and other harmful substances from entering the body through the intestinal mucosa [51].

\section{Conclusion}

In conclusion, the results obtained in the present study indicate that the increased in piglet growth with GOS supplementation was associated with the changes in expression of the genes and proteins involved in gut endocrine and barrier function, glucose transporter and immune status. Further study is needed to investigate the exact mechanisms by which GOS can promote intestinal development in suckling piglets.

\section{Additional file}

Additional file 1: Table S1. Primer sequences for quantitative real-time PCR analysis. (DOCX $17 \mathrm{~kb}$ )

\section{Abbreviations}

ADG: Average daily gain; BCA: Bicinchoninic acid; DAO: Diamine oxidase; DM: Dry matter; DP: Degree of polymerization; EGF: Epidermal growth factor; GAPHH: Glyceraldehyde phosphate dehydrogenase; GCG: Preproglucagon; GLP-2: Glucagon-like peptide-2; GLUT2: Glucose transporter type 2; GOS: Galacto-oligosaccharides; HE: Hematoxylin and eosin; IGF-1: Insulin-like growth factor 1; IGF-1R: Insulin-like growth factor 1 receptor; IL-

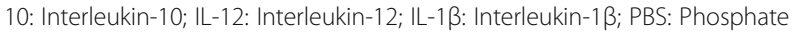
buffer saline; PBST: Phosphate buffer, saline with Tween-2;

PVDF: Polyvinylidene difluoride; SDS-PAGE: Sodium dodecyl sulfatepolyacrylamide gel electrophoresis; SGLT1: Sodium glucose co-transporter 1; 
SI: Small intestine; TGF- $\beta$ : Transforming growth factor- $\beta$; TLR4: Toll-like receptor 4; TNF-a: Tumor necrosis factor-a; ZO-1: Zonula occludens-1

\section{Acknowledgements}

The authors thank the National Center for International Research on Animal Gut Nutrition for financial support.

\section{Funding}

This study was supported by the National Key R\&D Program of China 2017 YFD0500505 and the Fundamental Research Funds for the Central Universities, China (KYZ201722).

\section{Availability of data and materials}

All data generated or analyzed during this study are available from the corresponding author upon reasonable request.

\section{Authors' contributions}

SYT and JW led the design and performance of the experiments, the analysis of the data, and the writing of the paper. HY participated in the animal feeding trials. JW and WYZ participated in designing the experiments and editing the paper. All authors read and approved the final manuscript.

\section{Ethics approva}

All animal care and procedures were approved by the Animal Care and Use Committee of Nanjing Agricultural University in compliance with the Regulations for the Administration of Affairs Concerning Experimental Animals (The State Science and Technology Commission of the People's Republic of China, 1988). The experiment was conducted at Nanjing Agricultural University.

\section{Consent for publication}

Not applicable.

\section{Competing interests}

The authors declare that they have no competing interests.

Received: 22 May 2018 Accepted: 5 September 2018

Published online: 18 October 2018

\section{References}

1. Collins CL, Pluske JR, Morrison RS, McDonald TN, Smits RT, Henman DL, et al. Post-weaning and whole-of-life performance of pigs is determined by live weight at weaning and the complexity of the diet fed after weaning. Anim Nutr. 2017:3:372-9.

2. Sundin $\mathrm{OH}$, Mendoza-Ladd A, Zeng M, Diaz-Arévalo D, Morales E, Fagan BM, et al. The human jejunum has an endogenous microbiota that differs from those in the oral cavity and colon. BMC Microbiol. 2017;17:160.

3. Buddington RK, Sangild PT. Companion animals symposium: development of the mammalian gastrointestinal tract, the resident microbiota, and the role of diet in early life. J Anim Sci. 2011;89:1506-19.

4. Pluske JR. Invited review: aspects of gastrointestinal tract growth and maturation in the pre-and postweaning period of pigs. J Anim Sci. 2016;94: 399-411.

5. Jacobi SK, Odle J. Nutritional factors influencing intestinal health of the neonate. Adv Nutr. 2012;3:687.

6. Tzortzis G. Functional properties of the second generation prebiotic Galacto-oligosaccharide (B-GOS). Agro Food Ind Hi Tec. 2009:20:43-6.

7. Vandenplas Y, Zakharova I, Dmitrieva Y. Oligosaccharides in infant formula: more evidence to validate the role of prebiotics. Brit J Nutr. 2015;113:1339-44

8. Boehm G, Moro G. Structural and functional aspects of prebiotics used in infant nutrition. J Nutr. 2008;138:1818S-28S.

9. Matsuki T, Tajima S, Hara T, Yahagi K, Ogawa E, Kodama H. Infant formula with galacto-oligosaccharides (OM55N) stimulates the growth of indigenous bifidobacteria in healthy term infants. Benef Microbes. 2016;7:453-61.

10. Monteagudomera A, Arthur JC, Jobin C, Keku T, BrunoBarcena JM, AzcaratePeril MA. High purity galacto-oligosaccharides enhance specific Bifidobacterium species and their metabolic activity in the mouse gut microbiome. Benef Microbes. 2016;7:247-64.
11. Maathuis AJ, Heuvel EG, Schoterman MH, Venema K. Galactooligosaccharides have prebiotic activity in a dynamic in vitro colon mode using a (13) C-labeling technique. J Nutr. 2012;142:1205-12.

12. Sosa N, Gerbino E, Golowczyc MA, Schebor C, Gómez-Zavaglia A, Tymczyszyn EE. Effect of Galacto-oligosaccharides: Maltodextrin matrices on the recovery of lactobacillus plantarum after spray-drying. Front Microbiol. 2016;7:584.

13. Varasteh S, Braber S, Garssen J, Fink-Gremmels J. Galacto-oligosaccharides exert a protective effect against heat stress in a Caco-2 cell model. J Funct Foods. 2015;16:265-77

14. Leforestier G, Blais A, Blachier F, Marssetbaglieri A, Davilagay AM, Perrin E, et al. Effects of galacto-oligosaccharide ingestion on the mucosa-associated mucins and sucrase activity in the small intestine of mice. Eur J Nutr. 2009:48:457-64.

15. Anthony JC, Merriman TN, Heimbach JT. 90-d oral (gavage) study in rats with galactooligosaccharides syrup. Food Chem Toxicol. 2006;44:819-26.

16. Pan XD, Chen FQ, Wu TX, Tang HG, Zhao ZY. Prebiotic oligosaccharides change the concentrations of short-chain fatty acids and the microbial population of mouse bowel. J Zhejiang Univ Sci B. 2009;10:258-63.

17. Moeser AJ, Klok CV, Ryan KA, Wooten JG, Little D, Cook VD, et al. Stress signaling pathways activated by weaning mediate intestinal dysfunction in the pig. Am J Physiol-Gastr L. 2007;292:G173-81.

18. Wang D, Xu S, Yan L, Fang Z, Che L, Xue B, et al. Recombinant porcine epidermal growth factor-secreting Lactococcus lactis promotes the growth performance of early-weaned piglets. Bmc Vet Res. 2014;10:171-82.

19. $\mathrm{Yu} \mathrm{LL}$, Zhong $\mathrm{XL}$. Evaluation of suitable reference genes for normalization of microRNA expression by real-time reverse transcription PCR analysis during longan somatic embryogenesis. Plant Physiol Biochem. 2013;66:20-5.

20. Livak KJ, Schmittgen TD. Analysis of relative gene expression data using real-time quantitative PCR and the $2^{-\Delta \Delta C T}$ method. Methods. 2001;25:402-8.

21. Hu CH, Gu LY, Luan ZS, Song J, Zhu K. Effects of montmorillonite-zinc oxide hybrid on performance, diarrhea, intestinal permeability and morphology of weanling pigs. Anim Feed Sci Tech. 2012;177:108-15.

22. Odle J, Lin X, Jacobi SK, Kim SW, Stahl CH. The suckling piglet as an agrimedical model for the study of pediatric nutrition and metabolism. Annu Rev Anim Biosci. 2014:2:419-44.

23. Alizadeh A, Akbari P, Difilippo E, Schols HA, Ulfman LH, Schoterman MHC, et al. The piglet as a model for studying dietary components in infant diets: effects of galacto-oligosaccharides on intestinal functions. Brit. J Nutr. 2016;115:605-18.

24. Widdowson EM, Crabb DE. Changes in the organs of pigs in response to feeding for the first $24 \mathrm{~h}$ after birth. Neonatology. 1976;28:261-71.

25. Hall RA, Widdowson EM. Response of the organs of rabbits to feeding during the first days after birth. Neonatology. 1979;35:131-9.

26. Heird WC, Schwarz SM, Hansen IH. Colostrum-induced enteric mucosal growth in beagle puppies. Pediatr Res. 1984;18:512-5.

27. Berseth $\mathrm{CL}$. Enhancement of intestinal growth in neonatal rats by epidermal growth factor in milk. Am J Physiol-Gastroint Liver Physiol. 1987;253:G662-5.

28. Xu RJ, Mellor DJ, Tungthanathanich P, Birtles MJ, Reynolds GW, Simpson HV. Growth and morphological changes in the small and the large intestine in piglets during the first three days after birth. J Dev Physiol. 1992;18:161-72.

29. Hoseinifar SH, Khalili M, Rostami HK, Ángeles EM. Dietary galactooligosaccharide affects intestinal microbiota, stress resistance, and performance of Caspian roach (Rutilus rutilus) fry. Fish Shellfish Immu. 2013;35:1416-20.

30. Yousaf MS, ljaz A, Ashraf K, Rashid MA, Hafeez A, Zaneb H, et al. Comparative effects of different dietary concentrations of $\beta$-galactooligosaccharides on growth performance, feed conversion efficiency and organs development in broilers. J Anim Plant Sci. 2016;26:1603-8.

31. Dube PE, Brubaker PL. Frontiers in glucagon-like peptide-2: multiple actions, multiple mediators. Am J Physiol Endocrinol Metab. 2007;293:E460-5.

32. Rowland KJ, Trivedi S, Lee D, Wan K, Kulkarni RN, Holzenberger M, et al. Loss of glucagon-like peptide-2-induced proliferation following intestinal epithelial insulin-like growth factor-1-receptor deletion. Gastroenterology. 2011:141:2166-75.

33. Donovan SM, Mcneil LK, Jiménez-flores R, Odle J. Insulin-like growth factors and insulin-like growth factor binding proteins in porcine serum and milk throughout lactation. Pediatr Res. 1994;36:159-68. 
34. Burrin DG, Stoll B, Guan X, Cui L, Chang X, Hadsell D. GLP-2 rapidly activates divergent intracellular signaling pathways involved in intestinal cell survival and proliferation in neonatal piglets. Am J Physiol-Endoc M. 2007;292:E281-91.

35. Leen J, Upadhyay C, Brubaker PL. S1621 Glucagon-Like Peptide-2 Increases Insulin-Like Growth Factor-1 mRNA Transcript Levels Through PI3-K/AKT in Murine Intestinal Subepithelial Myofibroblasts. Gastroenterology. 2009; 136:A-236.

36. Dubé PE, Brubaker PL. Frontiers in glucagon-like peptide-2: multiple actions, multiple mediators. Am J Physiol-Endoc M. 2007;293:E460-5.

37. Nofouzi K, Aghapour M, Hamidian G, Katiraee F, Stanford J, Ripley P. Oral administration of heat killed Tsukamurella inchonensis enhances immune responses and intestinal function in mice. Vet Med. 2016;61:681-8.

38. Bellinge RH, Liberles DA, laschi SP, O'brien PA, Tay GK. Myostatin and its implications on animal breeding: a review. Anim Genet. 2005:36:1-6.

39. Pieper R, Scharek-Tedin L, Zetzsche A, Röhe I, Kröger S, Vahjen W. Bovine milk-based formula leads to early maturation-like morphological, immunological, and functional changes in the jejunum of neonatal piglets. J Anim Sci. 2016;94:989-99.

40. Huygelen V, Vos MD, Prims S, Vergauwen H, Fransen E, Casteleyn C, et al. Birth weight has no influence on the morphology, digestive capacity and motility of the small intestine in suckling pigs. Livest Sci. 2015;182:129-36.

41. Monaco MH, Kashtanov DO, Wang M, Walker DC, Rai D, Jouni ZE, et al. Addition of polydextrose and galactooligosaccharide to formula does not affect bacterial translocation in the neonatal piglet. J Pediatr Gastr Nutr. 2011;52:210-6.

42. Mao X, Zeng X, Qiao S, Wu G, Li D. Specific roles of threonine in intestinal mucosal integrity and barrier function. Front Biosci. 2011;3:1192-200

43. Wijtten PJ, Meulen JV, Verstegen MW. Intestinal barrier function and absorption in pigs after weaning: a review. Brit J Nutr. 2011;105:967-81.

44. Chen H, Mao X, He J, Yu B, Huang Z, Zheng P, et al. Dietary fibre affects intestinal mucosal barrier function and regulates intestinal bacteria in weaning piglets. Brit. J Nutr. 2013;110:1837-48.

45. Laukoetter MG, Bruewer M, Nusrat A. Regulation of the intestinal epithelial barrier by the apical junctional complex. Curr Opin Gastroen. 2006:22:85-9.

46. Fukudome I, Kobayashi M, Dabanaka K, Maeda H, Okamoto K, Okabayashi T, et al. Diamine oxidase as a marker of intestinal mucosal injury and the effect of soluble dietary fiber on gastrointestinal tract toxicity after intravenous 5-fluorouracil treatment in rats. Med. Mol. Morphology. 2014;47:100-7.

47. Nielsen C, Lindholt JS, Erlandsen EJ, Mortensen FV. D-lactate as a marker of venous-induced intestinal ischemia: an experimental study in pigs. Int J Surg. 2011;9:428-32.

48. Akbari P, Braber S, Alizadeh A, Verheijden KA, Schoterman MH, Kraneveld $A D$, et al. Galacto-oligosaccharides protect the intestinal barrier by maintaining the tight junction network and modulating the inflammatory responses after a challenge with the mycotoxin deoxynivalenol in human Caco-2 cell monolayers and B6C3F1 mice. J Nutr. 2015;145:1604-13.

49. Bhatia S, Prabhu PN, Benefiel AC, Miller MJ, Chow J, Davis SR, et al. Galactooligosaccharides may directly enhance intestinal barrier function through the modulation of goblet cells. Mol Nutr Food Res. 2015;59:566-73.

50. Mereu A, Tedó G, Moeser AJ, Rimbach G, Ipharraguerre LR. Cromolynmediated improvement of intestinal barrier function is associated with enhanced piglet performance after weaning. BMC Vet Res. 2015;11:274-9.

51. Turner JR. Intestinal mucosal barrier function in health and disease. Nat rev Immunol. 2009;9:799-809.

\section{Ready to submit your research? Choose BMC and benefit from}

- fast, convenient online submission

- thorough peer review by experienced researchers in your field

- rapid publication on acceptance

- support for research data, including large and complex data types

- gold Open Access which fosters wider collaboration and increased citations

- maximum visibility for your research: over $100 \mathrm{M}$ website views per year

At $\mathrm{BMC}$, research is always in progress.

Learn more biomedcentral.com/submissions 